\title{
Prognostic Factors for Patients with Esophageal Cancer Receiving Definitive Radiotherapy Alone: A Retrospective Analysis
}

This article was published in the following Dove Press journal: Cancer Management and Research

\author{
Nan Jiang ${ }^{1,2, *}$ \\ Xiao-Lin $\mathrm{Ge}^{2, *}$ \\ Zhao-Yue Zhang ${ }^{2}$ \\ Jia Liu ${ }^{2}$ \\ Pei-Pei Wang ${ }^{2}$ \\ Xin-Chen Sun $\left(1 D^{1,2}\right.$ \\ Min Yang' \\ 'The First School of Clinical Medicine, \\ Nanjing Medical University, Nanjing, \\ Jiangsu, 210000, People's Republic of \\ China; ${ }^{2}$ Department of Radiotherapy, \\ Jiangsu Province Hospital, Nanjing, \\ Jiangsu, 210000, People's Republic of \\ China \\ *These authors contributed equally to \\ this work
}

Purpose: Intensity-modulated radiotherapy (IMRT) can improve the prognosis of patients with esophageal cancer. This study aimed to evaluate clinical factors relevant to the prognosis of patients with esophageal cancer who received intensity-modulated radiotherapy (IMRT) alone.

Patient and Methods: Data of 103 patients with pathologically confirmed esophageal cancer who were admitted to our hospital between October 2011 and November 2017 were retrospectively reviewed. All patients had squamous cell carcinoma. All patients received IMRT. Patients with stage I-IVA tumors were included to represent the real-world clinical practice. We performed univariate and multivariate analyses to identify prognostic factors for overall survival (OS) and progression-free survival (PFS). In univariate analyses, the Kaplan-Meier method was used to estimate OS and PFS for various subgroups. In multivariate analyses, hazard ratios were calculated.

Results: Single-factor analysis revealed that $\mathrm{T}$ stage $(\mathrm{P}=0.019), \mathrm{N}$ stage $(\mathrm{P}=0.047)$, and lesion length $(\mathrm{P}=0.000)$ were associated with the prognosis of esophageal cancer patients who received IMRT. Cox regression analysis revealed that $\mathrm{T}$ stage (odds ratio $[\mathrm{OR}]=4.68$; $\mathrm{P}<0.05), \mathrm{N}$ stage $(\mathrm{OR}=0.28 ; \mathrm{P}<0.05)$, and lesion length $(\mathrm{OR}=0.09 ; \mathrm{P}<0.05)$ were independent factors relevant to prognosis.

Conclusion: T stage, $\mathrm{N}$ stage, and lesion length influenced the long-term curative effects of IMRT for esophageal cancer and were prognostic factors for patients with esophageal cancer receiving definitive radiotherapy alone. The higher the stage and the longer the tumor, the lower the survival rate.

Keywords: esophageal neoplasms, prognosis, intensity-modulated radiotherapy, retrospective studies

\section{Introduction}

Esophageal cancer (EC) is the sixth most common cancer and the fourth leading cause of cancer-related deaths in China. ${ }^{1,2}$ The prognosis of EC is generally poor, and it can easily metastasize at early stages. Moreover, because patients often relapse after surgery, the 5-year survival rate of patients with EC is $17.1 \%{ }^{3}$ Thus, treating EC requires interdisciplinary management approaches.

While patients with early-stage EC can be treated with radical surgery, those with a locally advanced disease require the addition of neoadjuvant chemoradiotherapy (CRT) or perioperative chemotherapy. Such multifactorial approaches can improve locoregional control and the overall survival (OS) and progression-free

Xin-Chen Sun

Department of Radiotherapy, Jiangsu

Province Hospital, No. 300 of Guangzhou

Road, Gulou District, Nanjing, Jiangsu,

210000 , People's Republic of China

Tel +86 I3770662828

Email sunxinchen2012@I63.com 
survival (PFS). ${ }^{4}$ Although the introduction of multidisciplinary approaches has improved the outcomes of patients with EC, for many patients, comorbidities, poor performance status, and economic factors can preclude the use of combination approaches, especially those involving surgery or chemotherapy. ${ }^{5,6}$ Thus, in this study, we retrospectively analyzed the clinical data of 103 patients with EC who only received radiotherapy (RT) in our hospital and explored factors influencing patient prognosis.

\section{Methods}

\section{Patients}

Patients treated with radiotherapy for EC at the Department of Radiation Oncology at the Jiangsu Province Hospital between 2011 and 2017 were identified from a retrospective archival database of paper and electronic records. The inclusion criteria were (i) EC diagnosis based on pathological or cytological examinations, All patients had squamous cell carcinoma, (ii) Karnofsky performance status score $\geq 70$, (iii) no distant metastases, (iv) RT treatment alone, and (v) complete information on the curative effects of RT. The exclusion criteria were neoadjuvant radiotherapy, chemoradiotherapy, immunotherapy with irradiation, radiotherapy of metastases, previous or simultaneous malignancies, or incomplete data. Clinical stages were classified by the sixth edition of the American Joint Committee on Cancer (AJCC) staging system. The patient cohort for the final analysis included 103 patients (62 men, 41 women).

This study was conducted in accordance with the ethical standards of the World Medical Association Declaration of Helsinki and was approved by the ethics board at Jiangsu Province Hospital.

\section{Treatment}

All patients were treated with computed tomography (CT)planned intensity-modulated RT (IMRT) at the Jiangsu Province Hospital. The radiation field design included the primary tumor site and the mediastinal lymphatic drainage regions that were $3 \mathrm{~cm}$ cranial of the upper and caudal of the lower tumor borders. Coeliac lymph nodes were included for distally located tumors, while caudal cervical lymph nodes were included for tumors of the cervical or upper thoracic esophagus. If a boost was indicated, boost volume was defined with margins of $2 \mathrm{~cm}$ above and below the tumor borders. The patient's blood, routine liver and kidney function, and barium test results were reviewed after 3 weeks of treatment. Moreover, the change of tumor size were examined to reduce toxic and side effects of RT in accordance with the patients' individual needs.

\section{Follow-Up}

Patients were routinely examined with $\mathrm{CT}$ and endoscopy every 3-6 months for the first 2 years and every 6-12 months thereafter. The median follow-up period from the end of RT was 14 months for the entire cohort and 27 months for surviving patients. No patients were lost to follow-up.

\section{Statistical Analysis}

SPSS version 22.0 (SPSS Inc., Chicago, IL, USA) was used for data analysis. All survival times were calculated, starting from the date of the initial diagnosis. OS was defined as the time from the date of initial diagnosis to death. PFS was defined as the time from the date of initial diagnosis to local recurrence or occurrence of metastases depending on which event occurred first. Enumeration data are presented as relative numbers. In the univariate analysis, the Kaplan-Meier method was used to estimate OS and PFS for various subgroups. In multivariate analyses, hazard ratios were calculated. For all tests, P-values $<0.05$ were considered significant.

\section{Results}

In total, 103 patients were included in the analysis. Patient characteristics are listed in Table 1. Sixty-four patients $(62.1 \%)$ were aged $\geq 75$ years, and the median age was 77 years. Over $60 \%$ of the patients were male. Most patients had a locally advanced disease at the time of diagnosis. The location of the primary tumor (defined by its proximal edge) was the cervix in $3.8 \%$ of cases, upper thorax in $24.3 \%$ of cases, middle thorax in $41.7 \%$ of cases, and lower thorax/abdomen in $30.2 \%$ of cases. Sixty-seven patients $(65.0 \%)$ had a lesion length $>4 \mathrm{~cm}$. Eighty-three patients had gross tumor volumes (GTVs) $<64 \mathrm{~cm}^{3}$ (80.6\%). Sixty-four patients $(62.1 \%)$ received a total dose $>60$ Gy.

The median OS and PFS for the entire cohort were 15.0 and 13.0 months, respectively. The estimated 2- and 5-year survival rates were $34.3 \%$ and $18.2 \%$, respectively, for OS and $26.2 \%$ and $16.0 \%$, respectively, for PFS. Univariate analysis revealed that patient age was not associated with OS or PFS (Figure 1). Both tumor and nodal stage and lesion length had a significant influence on OS, with patients 
Table I Patient Characteristics

\begin{tabular}{|l|l|l|}
\hline Characteristic & & No. (\%) \\
\hline Age (year) & Median & $77(48-88)$ \\
& $<75$ & $39(37.9)$ \\
& $\geq 75$ & $64(62.1)$ \\
\hline Gender & Male & $62(60.2)$ \\
& Female & $41(39.8)$ \\
\hline Tumor stage & TI-T2 & $22(21.4)$ \\
& T3 & $17(16.5)$ \\
\hline Nodal stage (clinical) & T4 & $64(62.1)$ \\
\hline Lesion length (cm) & No & $77(74.8)$ \\
& NI & $26(25.2)$ \\
\hline GTV (cm ${ }^{3}$ ) & $\leq 4$ & $36(35.0)$ \\
& $>4$ & $67(65.0)$ \\
\hline Tumor location & $<64$ & $83(80.6)$ \\
& $\geq 64$ & $20(19.4)$ \\
\hline Total radiation dose (Gy) & $\leq 60$ & $4(3.8)$ \\
& $>60$ & $25(24.3)$ \\
& Cervical & $33(4 I .7)$ \\
& Upper thoracic & $39(30.2)$ \\
\hline & Middle thoracic & $64.9)$ \\
\hline
\end{tabular}

having T1/2 tumors or N0 status achieving a median OS of 25.9 and 29.6 months, respectively (Figures 2-4).

Cox regression analysis revealed that $\mathrm{T}$ stage (odds ratio $[\mathrm{OR}]=4.68 ; \mathrm{P}=0.000), \mathrm{N}$ stage (odds ratio $=$ $0.28 ; \mathrm{P}=0.013$ ), and lesion length (odds ratio $=0.09 ; \mathrm{P}=$ 0.000 ) were independent factors relevant to the prognosis of patients with EC who received IMRT, as shown in Table 2.

\section{Discussion}

EC presents obvious regional distribution worldwide, with associated morbidity and mortality clearly differing among countries. $^{7,8}$ China has a particularly high incidence of EC. ${ }^{9}$ Approximately $90 \%$ of all ECs present as squamous cell carcinoma, and the pathogenesis of the disease is believed to be related to the genetic background and acquired dietary habits. ${ }^{10}$

The morbidity rates of men and women with EC are approximately $30 / 100 \quad 000$ and 15/100 000, respectively. ${ }^{11,12}$ Most patients with EC are usually diagnosed at an advanced stage and thus lose the opportunity for a surgical option. In such cases, conservative definitive RT or CRT should be an effective method to treat

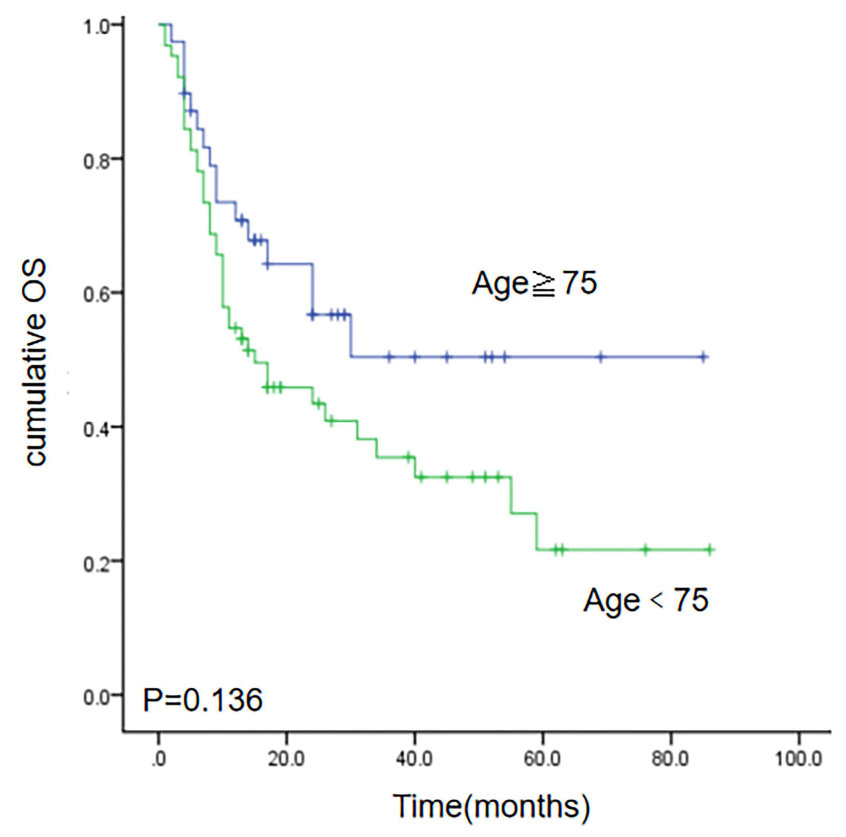

Figure I Kaplan-Meier estimates of overall survival (OS) for Age $\geq 75$ vs Age $<75$.

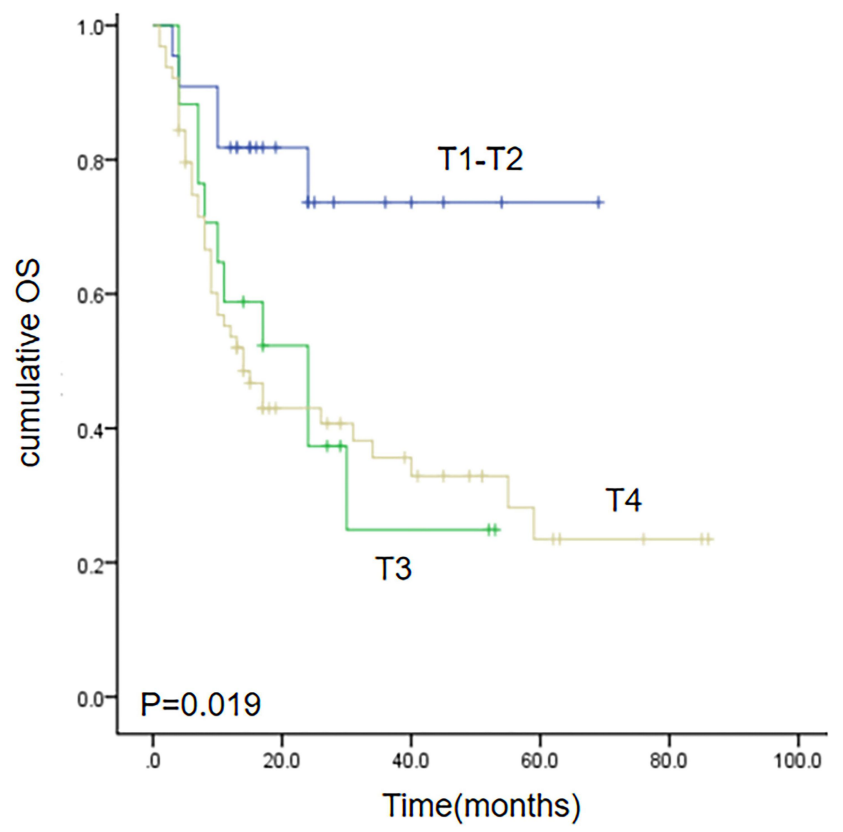

Figure 2 Kaplan-Meier estimates of overall survival (OS) for TI-T2 vs T3 vs T4.

patients. However, some people refuse chemotherapy because of financial reasons, complications, or psychological reasons. Such patients are typically treated with RT alone. RT and chemotherapy can drastically prolong survival and improve the quality of life. ${ }^{13-15}$ IMRT was developed in recent years and can significantly increase the dose of radiation to the tumor target area while reducing radiation exposure to the surrounding normal tissues. 


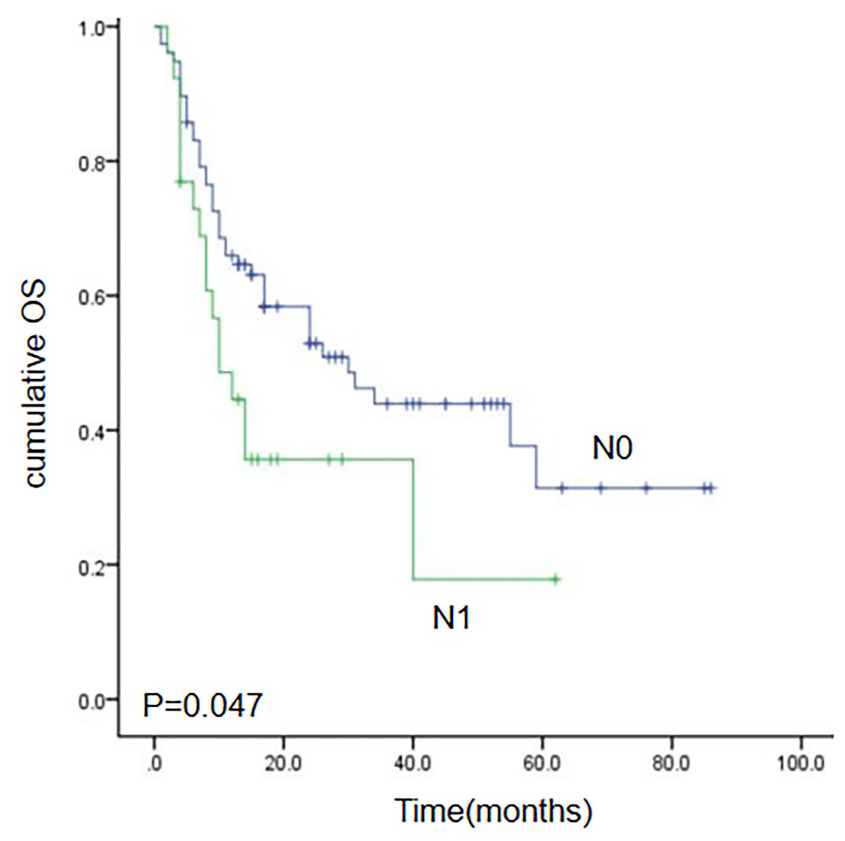

Figure 3 Kaplan-Meier estimates of overall survival (OS) for N0 vs NI.

This, in turn, improves the curative effects of radiation treatment while reducing related side effects. ${ }^{16}$ Under the condition that the radiation fields of the surrounding tissue are consistent with the shape of the target area, IMRT adjusts the irradiation intensity based on the threedimensional shape of the target area and incorporates the specific anatomical relationship between important organs and the target area.

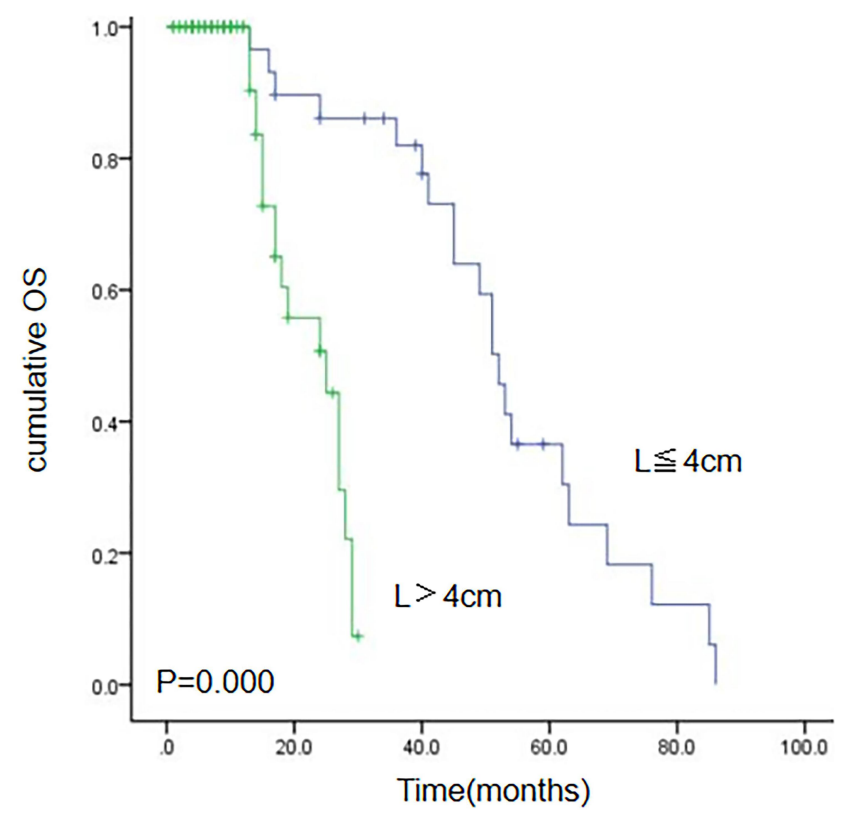

Figure 4 Kaplan-Meier estimates of overall survival (OS) for lesion length $\leq 4 \mathrm{~cm}$ vs $>4 \mathrm{~cm}$.
Table 2 Cox Regression Analysis of Independent Factors Relevant to Prognosis

\begin{tabular}{|l|l|l|l|}
\hline Factors & OR & $\mathbf{9 5 \%} \mathbf{~ C I}$ & $\mathbf{P}$ \\
\hline Age (year) & 1.248 & $0.54-2.85$ & 0.599 \\
\hline Gender & 0.430 & $0.15-1.16$ & 0.096 \\
\hline Tumor location & 0.674 & $0.26-1.73$ & 0.415 \\
\hline Lesion length (cm) & 0.095 & $0.03-0.3$ & 0.000 \\
\hline T stage & 4.680 & $2.05-10.71$ & 0.000 \\
\hline N stage & $0.28 I$ & $0.10-0.77$ & 0.013 \\
\hline Total radiation dose (Gy) & 1.438 & $0.70-2.92$ & 0.375 \\
\hline
\end{tabular}

Numerous factors, including the RT method, radiation dose, and clinical stage, influence the curative effect of RT for EC. ${ }^{17,18}$ However, results of clinical reports are not completely consistent, ${ }^{19,20}$ and to the best of our knowledge, no prediction model of the curative effects of RT for EC is available. Most reports on the curative effect of RT mainly focus on the choice of irradiation technique and the irradiation intensity, ${ }^{21}$ and such research on the influence of patients' clinical characteristics on the curative effect of RT is limited. Therefore, to explore the influence of their clinical characteristics on the curative effects of treatment, we retrospectively analyzed the data of recent EC patients who were admitted to our hospital and received IMRT. Among 103 patients who received RT, 36 patients survived $>2$ years and 67 patients died of tumor recurrence, distant metastasis, or related complications within 2 years of treatment. Thus, the 2-year survival rate was $35.0 \%$. Moreover, Cox regression analysis showed that an increasing T stage increased the odds of survival, while an increasing $\mathrm{N}$ stage and lesion length reduced the odds of survival, thus representing important prognostic factors for patients with EC who received IMRT.

The $T$ stage reflects tumor size and depth of invasion. In general, the higher the $\mathrm{T}$ stage, the larger the tumor and the deeper the tumor invasion. The tumor is large, the central area of the tumor is hypoxic. In this case, the cells in the center of the tumor are less sensitive to radiotherapy and therefore have a poor prognosis. The $\mathrm{N}$ staging reflects regional lymph node metastasis, The later the stage, the more extensive the regional lymph node metastasis.

Several other studies have shown that advanced T stage, ${ }^{22,23} \mathrm{~N}$ stage, ${ }^{24}$ and longer tumor length ${ }^{25}$ are strong indicators of a poor prognosis. However, these studies included either chemoradiotherapy or radiotherapy. In our study, all patients received definitive radiotherapy alone. 
This study had some limitations. First, this was a single-institution retrospective study with a relatively small sample size; the clinical relevance is relatively low. As such, our findings may not be generalizable to other clinical settings. Therefore, prospective studies with a larger sample size are needed to explore the relationship of $\mathrm{T}$ stages, $\mathrm{N}$ stages, lesion length, and other prognostic factors of EC with patient survival.

\section{Conclusion}

We established IMRT as a relatively effective treatment option for advanced EC, and the 2-year survival rate was approximately $35 \%$. T stage, $\mathrm{N}$ stage, and lesion length influenced the long-term curative effects of IMRT for EC. The higher the stage and the longer the tumor, the lower the 2-year survival rate.

\section{Abbreviations}

CT, computed tomography; EC, esophageal cancer; IMRT, intensity-modulated radiation therapy; OS, overall survival; PFS, progression-free survival.

\section{Data Sharing Statement}

The data that support the findings of this study are available from the corresponding author, upon reasonable request.

\section{Ethics Approval and Informed Consent}

The study was approved by the ethics committee of Jiangsu Province Hospital, the exemption of informed consent has been obtained (2019-SR-410). We guarantee that all patient data will be kept confidential.

\section{Acknowledgments}

I would like to show my deepest gratitude to my supervisor Dr. Min Yang and Dr. Xin-Chen Sun, respectable and responsible scholars, who have provided me with valuable guidance in every stage of writing of this thesis. Without their instructions and patience, I could not have completed my thesis. Nan Jiang and Xiao-Lin Ge are co-first authors for this study.

\section{Author Contributions}

All authors made substantial contributions to conception and design, acquisition of data, or analysis and interpretation of data; took part in drafting the article or revising it critically for important intellectual content; agreed to submit to the current journal; gave final approval of the version to be published; and agree to be accountable for all aspects of the work.

\section{Disclosure}

The authors declare that they have no conflicts of interest.

\section{References}

1. Chen WK, Sun R, Zheng H, et al. Cancer incidence and mortality in China, 2014. Chinese J Cancer Res. 2018;30(1):1-12. doi:10.21147/j. issn.1000-9604.2018.01.01

2. Chen WQ, Li H, Sun KX, et al. Report of cancer incidence and mortality in China, 2014. Zhonghua Zhong Liu Za Zhi. 2018;40 (1):5-13. doi:10.3760/cma.j.issn.0253-3766.2018.01.002

3. Allum WH, Stenning SP, Bancewicz J, et al. Long-term results of a randomized trial of surgery with or without preoperative chemotherapy in esophageal cancer. J Clin Oncol. 2009;27:5062-5067. doi:10.1200/JCO.2009.22.2083

4. Sjoquist KM, Burmeister BH, Smithers BM, et al. Survival after neoadjuvant chemotherapy or chemoradiotherapy for resectable oesophageal carcinoma: an updated meta-analysis. Lancet Oncol. 2011;12:681-692. doi:10.1016/S1470-2045(11)70142-5

5. Koppert LB, Lemmens VE, Coebergh JW, et al. Impact of age and comorbidity on surgical resection rate and survival in patients with oesophageal and gastric cancer. Br J Surg. 2012;99:1693-1700. doi:10.1002/bjs. 8952

6. Loffeld RJ, Dekkers PE. Experience with oesophageal cancer: a ten-year single centre study reflecting daily practice. ISRN Gastroenterol. 2013;2013:205417. doi:10.1155/2013/205417

7. Hongo M, Nagasaki Y, Shoji T. Epidemiology of esophageal cancer: orient to occident. Effects of chronology, geography and ethnicity. $J$ Gastroenterol Hepatol. 2009;24:729-735. doi:10.1111/j.14401746.2009.05824.x

8. Eslick GD. Epidemiology of esophageal cancer. Gastroenterol Clin North Am. 2009;38:17-25 vii. doi:10.1016/j.gtc.2009.01.008

9. Gao QY, Fang JY. Early esophageal cancer screening in China. Best Pract Res Clin Gastroenterol. 2015;29:885-893. doi:10.1016/j. bpg.2015.09.018

10. Trivers KF, Sabatino SA, Stewart SL. Trends in esophageal cancer incidence by histology, United States, 1998-2003. Int J Cancer. 2008;123:1422-1428. doi:10.1002/ijc.23691

11. Siegel RL, Miller KD, Jemal A. Cancer statistics, 2018. CA Cancer J Clin. 2018;68:7-30. doi:10.3322/caac.21442

12. Siegel RL, Miller KD, Jemal A. Cancer statistics, 2017. CA Cancer J Clin. 2017;67:7-30. doi:10.3322/caac.21387

13. Vellayappan BA, Soon YY, Ku GY, et al. Chemoradiotherapy versus chemoradiotherapy plus surgery for esophageal cancer. Cochrane Database Syst Rev. 2017;8:CD010511.

14. Liu B, Bo Y, Wang K, et al. Concurrent neoadjuvant chemoradiotherapy could improve survival outcomes for patients with esophageal cancer: a meta-analysis based on random clinical trials. Oncotarget. 2017;8:20410-20417. doi:10.18632/oncotarget.14669

15. Sasaki Y, Kato K. Chemoradiotherapy for esophageal squamous cell cancer. Jpn J Clin Oncol. 2016;46:805-810. doi:10.1093/jjco/hyw082

16. Mizowaki T. Intensity-modulated radiation therapy (IMRT). Nihon Rinsho. 2011;69(Suppl 5):412-417.

17. Fokas E, Weiss C, Rödel C. The role of radiotherapy in the multimodal management of esophageal cancer. Dig Dis. 2013;31:30-37. doi: $10.1159 / 000347170$

18. Serkies K, Badzio A, Jassem J. The role of radiotherapy in the management of esophageal cancer. Pol Merkur Lekarski. 2005;18:332-335. 
19. Wolf M, Zehentmayr F, Niyazi M, et al. Long-term outcome of mitomycin C- and 5-FU-based primary radiochemotherapy for esophageal cancer. Strahlenther Onkol. 2010;186:374-381. doi:10.1007/ s00066-010-2137-y

20. Geh JI, Bond SJ, Bentzen SM, et al. Systematic overview of preoperative (neoadjuvant) chemoradiotherapy trials in oesophageal cancer: evidence of a radiation and chemotherapy dose response. Radiother Oncol. 2006;78:236-244. doi:10.1016/j.radonc.2006.01.009

21. Verma V, Moreno AC, Lin SH. Advances in radiotherapy management of esophageal cancer. J Clin Med. 2016;5:E91. doi:10.3390/jcm5100091

22. Ariga H, Nemoto K, Miyazaki S, et al. Prospective comparison of surgery alone and chemoradiotherapy with selective surgery in resectable squamous cell carcinoma of the esophagus. Int J Radiat Oncol Biol Phys. 2009;75:348-356. doi:10.1016/j.ijrobp.2009.02.086
23. Teoh AY, Chiu PW, Yeung WK, et al. Long-term survival outcomes after definitive chemoradiation versus surgery in patients with resectable squamous carcinoma of the esophagus: results from a randomized controlled trial. Ann Oncol. 2013;24:165-171. doi:10.1093/annonc/mds206

24. Yin $\mathrm{H}$, Li D, Zhu C, et al. Factors relevant to the prognosis of patients with esophageal cancer who received intensity-modulated radiotherapy. Thoracic Cancer. 2018;9:1215-1219. doi:10.1111/ 1759-7714.12800

25. Cheng Y, Chen $\mathrm{H}$, Wu S, et al. Esophageal squamous cell carcinoma and prognosis in Taiwan. Cancer Med. 2018;7(9):4193-4201. doi:10.1002/cam4.1499

\section{Publish your work in this journal}

Cancer Management and Research is an international, peer-reviewed open access journal focusing on cancer research and the optimal use of preventative and integrated treatment interventions to achieve improved outcomes, enhanced survival and quality of life for the cancer patient.
The manuscript management system is completely online and includes a very quick and fair peer-review system, which is all easy to use. Visit http://www.dovepress.com/testimonials.php to read real quotes from published authors. 